\title{
Variations sur le thème de l'Américanité
}

\author{
Laura LÓPEZ MORALES \\ Universidad Nacional Autónoma de México
}

"Je dois rendre grâce à Dieu qu'on soit enfin venu me découvrir".

Entrée du 12 octobre 1492 d'un imaginaire journal intime de l'Amérique.

Edmundo O'Gorman, La invención de América: 1984, 15

Parler de l'Amérique, des Américains, de l'américanité, dans l'intention de nommer un continent, ceux qui l'habitent ainsi que leur sentiment d'appartenance à ce territoire comporte, pour commencer, une série d'ambiguïtés que nous ne pourrons que signaler dans ce travail. A cet effet, nous ferons un rapide survol sur le rapport entre l'idée de l'Amérique telle qu'elle fut conçue à l'origine par les Européens et la réalité qu'ils ont tenté de faire correspondre à leur rêve. Nous verrons ensuite, comment, surtout à partir du XVIIème siècle, se sont formés deux grands blocs marqués par le sceau culturel lié à l'identité du colonisateur, ce qui sera décisif pour leur développement respectif. Entre l'univers anglosaxon et la latinité brandie par Napoléon III, l'américanité empruntera deux voies différentes qui passent par la doctrine Monroe. Sur cette toile de fond, où se situe l'américanité des anglophones des E. U. et du Canada, celle des hispanophones et des lusophones et celle des Québécois, Français d'Amérique, donc aussi latins que ces latinoaméricains définis essentiellement par le métissage et le sous-développement? Il s'agit là de tout un programme qui demanderait un espace bien plus vaste que celui dans lequel j'aurai l'audace de l'approcher.

En effet, le discours autour du Nouveau Monde fut construit, au début, à partir de l'imaginaire, des conceptions et des modes de verbalisation des Européens qui après avoir découvert le continent en ont entrepris la conquête et la colonisation. ${ }^{2}$ Les chroniques et autres documents issus de ces pages historiques

\footnotetext{
${ }^{1}$ La traduction en français des sources en espagnol est nôtre.
} 
sont en quelque sorte devenus des paradigmes discursifs pour définir l'Amérique et l'américanité. C'est ainsi que, et tout le monde le sait, le Nouveau Monde fut d'abord connu sous le nom d' Indes Occidentales, et ce même après que, vers 1507 , les cartes géographiques commencent à associer le nom d'Americo Vespucci au continent découvert, plus tard baptisé de son nom. ${ }^{3}$ Une nouvelle ambigüité s'installe lorsque deux siècles après, on s'est mis des deux côtés de l'Atlantique à désigner du nom du continent les seuls Etats-Unis, récemment émancipés de la couronne britannique; c'est alors que le discours a commencé à opérer des glissements de sens qui demanderaient à être redressés ou, du moins, ce qui est en partie notre intention, à appeler un minimum de précisions quant au référent évoqué selon celui qui produit le discours. Il en va ainsi, par exemple, de ce que Jean Morency nous dit: "Pour le commun des mortels, est d'abord 'américain' ce qui relève des seuls Etats-Unis d'Amérique. On ne songera au reste du continent que par la suite, dans un sursaut de mauvaise conscience" (Morency, 1994: 9).

Or, si notre propos est d'approcher la manière dont nous tous, Américains au sens premier et plein du terme, nous sentons appartenir à ce continent, il devient indispensable d'éclaircir ce à quoi pense chacun de nous lorsqu'il est question de nous définir comme Américains, ce que représente cette identité, cette américanité que l'histoire a façonnée avec des éléments communs, mais aussi avec d'autres on ne peut plus dissemblables. Et puisque nous ne sommes pas d'accord avec le propos de Jean Morency, il nous faut jeter un coup d'oeil rapide aux enjeux sous-jacents à la découverte de cet espace géographique, le continent, et à l'ouverture d'une page historique nommée Indes Occidentales, Nouveau Monde et Amérique, mais dont l'idée fut au départ créée à l'image de son créateur, c'est-à-dire, l'Européen de la Renaissance. C'est encore Jean Morency, avec qui cette fois-ci nous sommes d'accord, qui dit à ce sujet:

[...] la découverte du Nouveau Monde ouvre des possibilités infinies, non plus seulement à l'intelligence et à la raison, mais aussi à l'imagination, ce qui explique sans doute son attrait sur les esprits, attrait qui survit encore de nos jours: elle apparaît comme la phase positive ou créatrice de la

${ }^{2}$ Dans 1492 , Jacques Attali fait une reconstruction minutieuse des expériences, connaissances et conditions du monde européen qui permirent un événement aussi capital pour la planète que l'arrivée de Colomb sur notre continent, date après laquelle, pour personne, rien ne serait plus comme avant.

${ }^{3}$ Ce fut le cartographe allemand Martin Waldssemüller qui, le premier, nomma Amérique le continent nouveau, dans son ouvrage Cosmographiae introductio cum quibusdam geometriae ac astronomiae principiis ad eam rem necessariis, insuper quatuor Aerici (sic) Vespucii navigationes, publié en 1507 (Cité par Litalien, 1993: 46) 
Renaissance, qui vient se superposer à l'effort rationnel de déconstruction de l'Ordre ancien (Morency, 1994: 10).

Pour sa part, Michel Morin estime que:

[...] la découverte de l'Amérique impliquait une sortie de l'histoire européenne en même temps que l'inauguration d'un nouveau rapport à l'espace et au temps [...] la conscience de ces nouveaux "Américains" ne s'est pas trouvée accordée à cette rupture et n'a pas pu immédiatement intérioriser la discontinuité; elle a plutôt vécu, sur le mode du fantasme, une apparence de continuité qui s'en trouvait dès lors désaccordée à l'expérience réelle, celle d'un nouvel espace, d'une nouvelle géographie, de nouveaux climats, de nouveaux types de sociétés humaines (Morin, 1982: 163-164).

Dans ce processus, deux voies se sont offertes qui ont conduit soit à l'adaptation des nouvelles circonstances à l'image du modèle, pris comme une sorte d'archétype, soit à prendre le modèle comme point de départ d'un projet assumé de manière indépendante, ce qui supposait adapter le paradigme aux nouvelles circonstances. Autrement dit, il y avait le choix entre l'imitation ou l'originalité. ${ }^{4}$ Il n'est pas inutile de rappeler ces conditions de départ car, conjuguées à la spécificité des cadres préexistants à l'arrivée des Européens, elles expliqueront en partie la différence de destinées entre les deux grands blocs que sont à présent l'A mérique du Nord et l'A mérique latine. Nous utilisons ces deux expressions qui sont du domaine publique, mais nous voudrions néanmoins signaler qu'elles supposent aussi quelques imprécisions: pour commencer, le Mexique fait partie de l'Amérique du Nord au même titre que les Etats Unis et que le Canada; d'un autre côté, pour ce qui est de la revendication de la latinité, par opposition au patrimoine anglo-saxon définissant majoritairement les Etats-Unis et le Canada, il faudrait associer le Québec à ce fond latin dont provient la France, comme la province canadienne n'a cessé de le faire tout au long de son histoire.

Mais revenons donc au point de départ évoqué plus haut. Comment, en fonction des deux voies possibles empruntées par les Européens dans la construction de ce Nouveau Monde, en sont-ils arrivés à faire incarner des rêves qui parfois ressemblaient à l'utopie qui avait guidé leur aventure, mais d'autres fois ils ont continué de poursuivre leur quête impossible? Selon Edmundo O'Gorman, l'Espagne et le Portugal, tout en s'appuyant sur les croyances, les moeurs et

${ }^{4}$ À cet égard la lecture de Genèse des nations et cultures du Vouveau Monde de l'historien québécois Gérard Bouchard est très éclairante par l'approche comparatiste qu'il fait de l'évolution des différents groupes établis sur tout le continent américain. 
les systèmes institutionnels de l'Europe, auraient choisi la première formule -celle de l'imitation -, tandis que l'Angleterre aurait opté pour la seconde, celle de l'originalité. Et la différence dans les résultats s'est vue accentuée par le fait que, d'après l'historien mexicain, pour les Anglais, le

\begin{abstract}
[...] processus de transformation [fut] encouragé par le sentiment que les nouvelles terres n'étaient pas un cadeau de la Providence pour accroître la puissance et la richesse de la métropole, mais l'occasion d'exercer, sans les empêchements traditionnels, la liberté religieuse et politique et ainsi donner libre cours à l'effort et au génie personnels. C'est ainsi que, dans un cadre bigarré de crovances, de moeurs et de tempéraments raciaux, les groupes qui se sont installés ont fondé, chacun à sa manière, la Nouvelle Jérusalem de sa préférence (O'Gorman, 1984: 157).
\end{abstract}

L'homme de la Renaissance a cru que le Nouveau Monde était la véritable possibilité de faire incarner ses vieilles utopies, de Platon à Thomas Moro, dont la filiation est claire jusqu'à Vasco de Quiroga et Bartolomé de las Casas. Cette tendance s'est en quelque sorte perpétuée dans les rêves et les entreprises des figures de proue ou du petit peuple anonyme, qui traversèrent l'océan poussés par le mirage du renouveau. Plus tard, la liste devait inclure des personnages nés déjà sur le continent: les Simón Bolivar, José Martí, Augusto César Sandino, José Vasconcelos qui, pour la plupart, misaient sur la dimension métisse de l'Amérique comme support et source essentiels de la richesse du continent. ${ }^{5}$ Il faudrait ajouter que, depuis quelques décennies, ce besoin d'asseoir un profil identitaire sur les composantes raciales et culturelles d'une histoire commune a conduit les peuples latinoaméricains à la récupération de l'apport des peuples africains dans la construction de l'identité non seulement antillaise mais plus largement américaine. En effet, la prise de conscience de ce qu'on a appelé la troisième racine, les deux autres correspondant aux peuples indiens préhispaniques et aux colonisateurs européens, n'est que la reconnaissance de la présence, à différents niveaux, des traces raciales et culturelles laissées par les peuples noirs dans le métissage américain. En somme, en dehors d'autres traits qui malgré tout peuvent les rapprocher, le dénominateur commun entre les colonies anglo-saxones, espagnoles et portugaise d'Amérique est bien la présence des populations africaines qui, en dépit de leur condition d'esclaves, ont marqué de leur sceau indélébile les nouvelles cultures nées des brassages

\footnotetext{
${ }^{5}$ Nous devrions ouvrir ici une sorte de sous-chapitre sur le multiethnisme actuel tant au Canada qu'aux Etats Unis, situation qui entraîne inévitablement le brassage des divers groupes et donc un métissage racial et culturel.
} 
entre colonisés et colonisateurs.

Pour en revenir à la formation progressive des deux blocs culturels américains, ceux qui ont étudié les premières chroniques des colonisateurs espagnols et portugais, d'une part, et celles des anglosaxons et des français, de l'autre, s'accordent pour dire que les respectives perceptions du Nouveau Monde étaient assez différentes. Les premiers insistent sur l'expérience de l'Européen devant l'exubérance de la nature, sur leur éblouissement face aux richesses de ces contrées ainsi que devant l'état de pureté des habitants de ces terres; on y voit déjà poindre les élans lyriques jouant dans la construction du mythe du bon sauvage ${ }^{6}$ et, peut-être aussi la cupidité mal déguisée de certains, derrière l'esprit rédempteur des autres. Quant aux Anglosaxons, le caractère imposant du nord du continent, avec ses bois, ses montagnes et ses grandes étendues enneigées, est peut-être aussi important qu'un autre aspect de la nouvelle réalité et qui posera conflict: la présence de l'Autre, ${ }^{7}$ vécue plutôt comme une menace, au point où la lutte entre le Blanc et l'Indien demeurera un sujet récurrent dans la littérature nordaméricaine, des États Unis et du Canada. Avec sa chronique True Relation of Occurrences and accidents in Virginia, John Smith encourage une tradition, reprise plus tard par Waldo Emerson et par Henry Thoreau, en ce qui concerne la nécessité de détruire une civilisation afin de permettre la naissance d'une autre meilleure; il va de soi que la culture destinée à la disparition était celle des groupes amérindiens au bénéfice de l'incarnation des rêves des Européens. Nous voyons ainsi se dessiner une toute autre attitude car, sans prétendre passer sous silence les atrocités et les abus commis par Espagnols et Portugais en Amérique, il faut tout de même souligner que leur politique de colonisation tenait en bonne partie de l'esprit de croisés et qu'ils ont cherché, peut-être pas toujours avec le succès qu'ils auraient escompté, à gagner des âmes pour la religion et à incorporer les populations au projet des nouvelles villes. En effet, on ne souligne peut-être pas assez comment la différence des religions, catholicisme dans les colonies ibériques et protestantisme dans l'espace anglo-saxon, représentait en soi tout un bagage mental et culturel qui, en bonne mesure, déciderait et marquerait le projet social et le développement des différentes colonies américaines. Le syncrétisme qui se produit entre le catholicisme et les religions préhispaniques est le corollaire d'un certain métissage racial et

${ }^{6} C f$. Rosa Beltrán, América sin americanismos, 1996, p. 13. Dans son étude Imagología del bueno y del mal salvaje, Juan A. Ortega y Medina approfondit l'analyse des images dégagées des textes parlant des habitants du Nouveau Monde écrits par des Européens du xVlème au XIXème siècles.

${ }^{7}$ Pour mieux saisir cette problématique, la lecture du texte célèbre de Todorov (1982) s'avère très utile. 
n'est pas étranger à l'émergence et à l'épanouissement d'un art et d'une pensée baroques. Par contre, la foi calviniste des colons anglo-saxons les prédisposait à se prendre pour les élus venus s'installer dans ce Nouveau Monde où ils avaient à jouer le rôle de maîtres et de protecteurs.

Par ailleurs, sans entrer dans le détail des trajectoires suivies localement par les envoyés des différentes puissances européennes, il nous faudrait tenir compte du fait que, dans le processus de constitution d'une conscience d'appartenance, d'une conscience identitaire, trois facteurs sont déterminants: un territoire, un peuple et un Etat. Or, c'est la délimitation d'un espace, dont l'Etat est garant, qui assure aux groupes humains la possibilité des diverses manifestations culturelles qui légitiment leur être. Dans ce sens, le nationalisme peut être conçu comme l'aboutissement d'un lent processus inscrit dans l'histoire des peuples selon lequel le sentiment d'appartenance, la pratique de certaines traditions —dont la religion-, de coutumes et d'une langue qui se serait imposée à d'autres, constituent le consensus d'une culture liée à un espace géographique. Michel Morin, qui a étudié particulièrement cette problématique dans le cas de la colonie française du Canada, affirme que "la répétition en Amérique de la vieille France sous le nom parodique de Nouvelle France n'a rien à voir avec l'inauguration d'une civilisation nouvelle [...] elle n'est que l'aboutissement, la pleine réalisation d'une logique schizophrénique" (Morin, 1982: 164-165) qui, plus tard, aura son rôle à jouer dans l'impossibilité de faire coïncider les trois instances évoquées plus haut, à savoir: territoire, peuple et Etat, indispensables à l'émergence d'une culture et donc à la construction d'une identité. A la différence des Etats Unis et du Canada anglophone, ainsi que des ex-colonies ibéroaméricaines, le rapport que la société franco-canadienne entretient avec l'Etat, considérant celui-ci comme instance déterminante de légitimation de l'identité, a toujours été très conflictuel du fait que ces pays, après la rupture avec les anciennes métropoles, ont donné naissance à de véritables Etats, ce qui ne fut pas le cas pour les colons d'origine française. Voilà ce qu'en dit Michel Morin qui s'est largement penché sur la question:

[...] la colonisation française en Amérique du Nord [...] quoique couvrant, à une certaine époque, un très vaste territoire qui s'étendait du nord au sud du continent, n'a jamais été aussi sy stématique que la colonisation anglaise. La métropole n'y a jamais accordé qu'une attention distraite et un intérêt fort limité. Ainsi fortement laissée à elle même, cette colonisation réussit à constituer un empire assez impressionnant grâce aux efforts des explorateurs, des coureurs des bois, et grâce aux liens que ceux-ci réussissaient 
à entretenir avec les Indiens. Son cours fut sans doute ainsi plus "naturel" [si nous pouvons nous permettre un tel mot], que celui de la colonisation anglaise: par là nous entendons qu'il fut moins commandé de l'extérieur et moins soumis aux normes et exigences de la métropole. C'est à ce relatif abandon par la métropole plutôt qu'à quelque trait "racial" ou "national" des Français que nous attribuerions la facil ité des contacts noués avec les Indiens en même temps que le très fort attrait que ceux-ci ont exercé sur les Français. La fragilité de l'empire français d'Amérique s'expliquerait ainsi par ce relatif effacement de l'Etat (Morin, 1982: 116).

Mais si la province francophone d'Amérique devint ainsi une sorte d'enclave française coupée de l'ancienne métropole, il ne faut pas croire que les diverses succursales américaines des puissances européennes se connaissaient bien entre elles. En réalité, les rivalités définissant bien avant les relations entre l'Espagne et l'Angleterre alimentèrent les préjugés conduisant depuis le XVIème siècle à une méconnaissance réciproque entre les colonies ibériques et anglosaxonnes du Nouveau Monde, le tout favorisé par une absence presque totale d'information sur ce qui se passait d'une part et de l'autre. Pour José Fuentes Mares, "l'information [aux Etats Unis] sur la portion ibéroaméricaine du continent était maigre dans les élites - à plus forte raison chez le petit peuple - [pour qui ] le monde hispanique était la région tropicale exotique, assombrie par une dictature médiévale et peuplée par un mélange de races étrangères à la culture anglaise" (Fuentes, 1984: 4). Nous pouvons ainsi imaginer que, très vraisemblablement, il en était de même, et peut-être davantage, chez les sujets britanniques du Canada. Nous voudrions néanmoins signaler que la presse étatsunienne a joué un rôle important dans l'intérêt des anglo-saxons pour l'espace ibéroaméricain; aussi faut-il faire mention, à titre de curiosité, des quelques cas d'hommes à l'esprit aventurier qui ayant eu vent des guerres d'indépendance des colonies ibériques ou de résistance à d'autres interventions étrangères, ont rejoint soit les insurgés, soit les troupes françaises envoyées par Napoléon III, comme ce fut le cas de certains franco-canadiens nostalgiques ${ }^{8}$ qui caressaient l'espoir de voir se rétablir la présence de la France en Amérique (!!!).

\footnotetext{
${ }^{8}$ Faucher de Saint-Maurice, avocat et militaire québécois dont l'histoire a quelque chose de légendaire, décide de se joindre à l'armée francaise qui fait la guerre aux forces de Juárez. Au bout d'un mois de voyage, Faucher parvient à être nommé officier d'ordonnace et c'est à ce titre qu'il prend part à onze batailles et deux sièges, à Oaxaca et à Saltillo. Condamné à la peine capitale, il est épargné grâce à l'échange d'un officier ennemi. Dans sa vision manichéenne, il vouera un culte presque sacré à la figure de Maximilien et taxera de sanguinaires les "guerrilléros" qui s'opposaient à l'invasion étrangère (Provencher, "Introduction", Contes et récits, Faucher Saint Maurice, 1977: 9-27).
} 
Par ailleurs, s'il est bien vrai que l'on encourt des risques de simplification à employer certains termes pour évoquer des référents qui imposent des nuances, il n'y en a pas moins à les éviter car, pour guider la réflexion et l'analyse, il nous faut nous appuyer sur des concepts intégrateurs, tout en veillant à ce qu'ils ne tournent à la totalisation simplificatrice. Il en va ainsi de la notion de latinité qui, juxtaposée à celle d'américanité, en est venue à agglomérer notamment les anciennes colonies hispaniques et lusophone. Déjà la revendication, au XIXème siècle, de la latinité pour distinguer ce bloc face au géant anglo-saxon naissant, représente en soi tout un programme de réflexion. C'est curieusement Napoléon le petit qui, à peine au lendemain de la perte de plus de la moitié du territoire mexicain au profit des Etats-Unis, fait appel à ces racines latines de l'Amérique ibérique cherchant à invoquer un lien qui rassemble les jeunes républiques, qui nourrisse un idéal d'union entre elles pour parer aux tendances expansionnistes de la puissance nord-américaine. Il va sans dire que l'encouragement de l'empereur français n'était pas non plus tout à fait désintéressé, et nous, les Mexicains, en savons quelque chose. Ceci dit, c'est encore une fois le discours élaboré ailleurs, en Europe, qui continue d'orienter la construction de l'identité des habitants du Nouveau Monde. Les mobiles de Napoléon III, lorsqu'il avance la notion de latinoaméricanité, obéissaient au projet de relier les jeunes républiques américaines au monde de la modernité, tout en les soustrayant à l'emprise des E. U. La France d'alors vivait dans des conditions aptes à nourrir le rêve d'une présence culturelle, et si possible économique et politique, sur le vaste territoire récemment émancipé du joug ibérique. C'est ainsi que le drapeau de la latinité l'autorisait à revendiquer une parenté vis-à-vis des peuples qu'il fallait protéger des ambitions des E. U.

Si les rêves napoléonniens ont échoué sur le plan politique, l'influence culturelle et idéologique de la France, marquante depuis la fin du XVIIIème siècle sur toute l'Amérique ibérique, s'est accentuée au cours de la seconde moitié du XIXème siècle. Néanmoins, les premiers symptômes qui entament le déclin de cette emprise commencent à se manifester à la faveur de l'étendard "panaméricaniste" qui faisait son petit chemin depuis 1823 , date du discours prononcé par le président des E. U. James Monroe. Dans ce texte le pragmatisme de la jeune puissance remplace l'idéalisme culturel français, et slogan pour slogan, pour combattre la référence au fond latin dans la construction de l'identité des habitants de l'Amérique, Monroe trouve mieux en revendiquant le droit des Américains à gérer leur destin, sans l'intervention des puissances européennes; c'est l'origine de la devise de "l'Amérique pour les Américains".

Il n'est peut-être pas sans intérêt de signaler un détail curieux: dans sa référence à la latinité, Napoléon III n'avait pas songé en premier au bas Canada de l'époque, c'est-à-dire le Québec actuel, dont il pouvait à plus juste titre 
réclamer les racines latines. C'est plus récemment que cette portion de l'identité québécoise a été revendiquée par ce peuple obéissant au besoin de préserver son autonomie culturelle face au rouleau compresseur que représente le modèle anglo-américain. En effet, il devient de plus en plus fréquent que ces Français d'Amérique invoquent le fonds latin qui leur revient de leur ancienne métropole pour établir un pont les reliant au bloc latinoaméricain. Une appréciation hâtive de ces données historiques pourrait néanmoins conduire à des conclusions fausses; nous voulons dire par là que de par ce lointain ancêtre commun aux Québécois et aux Latinoaméricains que serait la Rome impériale et sa culture, le référent auquel renverrait l'américanité serait le même pour les uns et pour les autres. Or, ce n'est vrai qu'en partie, comme nous tentons de le montrer à travers ces quelques réflexions, car les différences sont pour l'instant plus évidentes. Mais rien ne dit que ces origines latines ne puissent devenir, avec d'autres soucis communs, une véritable plateforme pour l'entente de ces peuples. Dans ce sens, on ne peut pas perdre de vue le parcours décrit par le peuple francophone en Amérique continentale, largement marqué par le voisinage avec les groupes migratoires anglo-saxons avec lesquels les rapports ont souvent été tendus. Et, compte tenu du but de notre approche, il convient également d'observer la trajectoire décrite par le gros bloc des colonies ibéroaméricaines qui, tout en inscrivant dans leur profil les traces de leur histoire particulière, offrent un fond commun et aspirent à des idéaux comparables. Dans un cas comme dans l'autre, leur sentiment d'appartenance à l'Amérique s'en trouve marqué de manière fort différente.

Selon les époques, intellectuels, philosophes et artistes ont cherché à saisir l'identité américaine en fonction de critères qui, soit par opposition, soit par filiation, privilégiaient ou méconnaissaient l'une de ses composantes culturelles. Ces perceptions pouvaient mettre en valeur les racines européennes - dont la latinité- ou, au contraire, préhispaniques; il arrivait aussi que l'on souligne plutôt le contraste vis-à-vis de la culture anglo-saxonne du nord du continent ou encore qu' on perçoive l'Amérique comme "l'espace cosmique de la cinquième race [... défini par ] sa bâtardise fondatrice" (Chiampi in Lezama, 1993: 9).

Des hommes tels que Pedro Henríquez Ureña, Alejo Carpentier, José Vasconcelos ou José Lezama Lima, parmi les plus connus, ont insisté sur l'idée d'une Amérique dont la marque distinctive était le métissage, dont l'unité culturelle était le résultat de sa continuité historique. Pour René Dépestre, ${ }^{9}$ il revient surtout à Carpentier le mérite de conjuguer et d'équilibrer les diverses composantes de cette identité que d'autres avaient exaltées séparémment.

${ }^{9}$ Dans sa révision des figures, des éléments et des moments clé dans la construction de l'identité ámericaine, Depestre souligne les apports de Carpentier et d'autres écrivains et intellectuels. 
Au-delà de tout compartimentage racial, il accueille les voix de Blancs et de Noirs, de Mulâtres et d'Indiens ouvrant ainsi la voie à d'autres représentants de la sève rénovatrice de l'américanité. Nicolás Guillén, Pablo Neruda, Jorge Amado, Juan Rulfo, Julio Cortázar, Gabriel García Márquez et beaucoup plus d'autres s'intègrent à ce choeur polyphonique.

Comme nous l'avons déjà avancé, c'est la similitude des expériences historiques qui décide de l'unité culturelle globale de l'Amérique latine car l'idée et le sentiment d'appartenance ne pouvaient pas être les mêmes dans les colonies où, comme ce fut le cas pour ce que deviendraient les Etats-Unis et le Canada, le peuplement des nouvelles terres fut majoritairement le fait de groupes d'immigrants européens. L'autre cas de figure déjà évoqué fut celui des colonies qui choisirent d'imposer, de répéter leurs modèles culturels d'origine sur des peuples possédant déjà des civilisations souvent très avancées. L'espace, en tant qu'instance d'appartenance à une culture, sera donc perçu très différemment par les uns et par les autres, du fait du transfert pur et simple des schémas organisationnels à un nouveau contexte, ou de la nécessité de tenir compte de ce qui existait sur place et, surtout, du projet de bâtir une nouvelle société avec ou en dépit des protagonistes qui les avaient précédés sur ces terres.

L'américanité des colons français et britanniques était liée à ce que, nous l'avons déjà dit, cet immense territoire pouvait incarner comme possibilité de façonner de vieux rêves, comme espace permettant de re-naître et de re-commencer une nouvelle vie. Ceci contrastait avec le projet des Espagnols et des Portugais qui y ont vu le défi de s'intégrer à d'autres groupes possédant déjà leurs propres structures sociales, leur vision du monde et, en tout cas, qu'il fallait inclure dans tout nouveau projet social, même si c'était sous prétexte de les civiliser et de les évangéliser. Il n'est pas inutile de rappeler que très tôt les consignes dictées par la couronne espagnole aux colonisateurs expriment bien une volonté de rencontre avec l'autre; ainsi le frère Nicolás de Ovando, envoyé à Saint Domingue en 1502, est prié de chercher à "ce que quelques chrétiens épousent des Indiennes et quelques chrétiennes en fassent autant avec des Indiens" De même, on sait que dès le début de la colonisation, les Espagnols ont fondé bon nombre de collèges et d'écoles destinés à l'instruction des Indiens.

Ainsi, même si l'américanité des colonies ibéroaméricaines a comporté au début, comme nous l'avons signalé, sa dose de rêve de renaissance — dans les deux sens du terme - elle a très vite dû s'adapter et se construire sur la base d'une réalité humaine tout autre: l'existence insoupçonnée de civilisations développées et souvent hautement raffinées n'a pas manqué d'émerveiller les

\footnotetext{
${ }^{10} \mathrm{C}$ 'est à cette époque que remonte le premier arrivage de femmes espagnoles au Nouveau Monde.
} 
colonisateurs européens. Au fur et à mesure que le brassage racial et culturel se produisait, que les nouveaux arrivés et leurs descendants apprivoisaient ces contrées, germait une nouvelle conscience d'appartenance à ces terres, tandis que la conquête accomplie par les Européens dans le nord du continent, une fois les Indiens chassés, parqués ou exterminés, représentait plutôt une victoire sur eux-mêmes face au défi lancé par ces énormes étendues qui alimentèrent leur besoin de renouveau, d'enracinement.

Nous l'avons souligné déjà à maintes reprises, les mobiles guidant les flux migratoires de l'Europe vers le Nouveau Monde avaient peut être un dénominateur commun lié aux idéaux de la Renaissance. Néanmoins, face à une réalité concrète mais multiple, les réactions ont également assumé diverses formes, d'autant plus que ces immigrants incarnaient eux aussi des types sociaux assez différents. On pourrait en distinguer au moins deux, qui ont fait l'objet d'études tant du point de vue historique et sociologique, que dans une perspective littéraire. Pour Michel Morin, autant que pour Jean Morency et pour Christian Morissonneau, les colons de l'Amérique du Nord, anglais et français, incarnaient d'une part le type social ou sédentaire, et de l'autre le type asocial ou nomade. Au premier groupe appartiendraient ceux qui deviendront des agriculteurs, qui encourageront la vie en groupe et contribueront à la naissance et à la croissance des villes; le second serait constitué par la race des coureurs des bois, des explorateurs, des aventuriers, des êtres errants dont la conscience des limites territoriales n'a de frontières que celles imposées par le hasard des déplacements. Dans la littérature francophone du Canada, et Jean Morency étend précisement son analyse de ces profils à la littérature de Etats-Unis, il existe d'innombrables exemples des deux types; pour ce qui est du nomadisme, nous pourrions même distinguer, surtout au cours de notre siècle, une variante assez curieuse: la quête d'une terre promise, le rêve de liberté et de renaissance qui dictent le départ des premiers colons vers les terres américaines, seront vécus à nouveau par certains de leurs descendants canadiens en direction des Etats-Unis sous l'effet de ce qu'on connaît comme le "rêve américain", qui consiste en la représentation du paradis de la modernité, du développement et de la liberté. Ainsi parler de l'Amérique égal Etats-Unis, revient aujourd'hui pour les Canadiens à ce qu'était autrefois pour les Européens parler du Nouveau Monde, c'est-à-dire, de tout le continent américain comme l'invocation d'un mirage de terre promise. Et lorsqu'ils revendiquent leur américanité c'est dans la mesure où ils peuvent faire leur cet imaginaire mythique des premiers colonisateurs du nord du continent où la marque essentielle est aux couleurs anglo-saxonnes. ${ }^{11}$

\footnotetext{
"Jean-Francois Chassay dans $L$ 'ambigüité américaine développe ce glissement de sens en disant que: "d'une définition territoriale, le mot 'Amérique' est passé au symbolique: mythe de la nouveauté et de la découverte" (1995: 19).
} 
Cette identification commence tout de même à présenter quelques fissures, du moins dans l'espace littéraire à en juger par ce que Gilles Thérien nous dit dans son essai "La littérature québécoise, une littérature du Tiers-monde?" (Thérien, 1986: 12-20). Nous pourrions même associer à cet état de "crise" d'identité les nouvelles données du panorama multiethnique et multiculturel que nous offre le Canada actuel et dont Neil Bissoondath, dans son Marché aux illusions (1995), brosse un tableau fort révélateur et intéressant.

Je continue de me poser la question, je dirais même plusieurs questions rattachées justement à la direction dans laquelle je voulais mener ma recherche de ce qu'étaient dans l'imaginaire des Canadiens en général, et des francophones en particulier, les autres américains, amérindiens, européens, africains et métis. De quelle manière se représentaient-ils leur appartenance à ce Nouveau Monde qu'ils partageaient avec d'autres peuples comparables et à la fois différents? Au cours de mes lectures, $j$ 'ai découvert des témoignages fort intéressants d'un courant états-unien que Jủan Ortega y Medina nomme "monroisme archéologique" et qui n'est autre que le versant culturel de la doctrine du panaméricanisme évoquée plus haut. Les premières traces de l'intérêt des colons anglais pour entrer en contact avec les colonies hispaniques remontent au XVIIème siècle à en juger par la version en espagnol que le précheur protestant Cotton Mather fait du catéchisme calviniste destiné à ses voisins les colons espagnols et aux Indiens: La Fe del Christiano anbiada a los espagnoles, en Veinte y Quatro Lecciones, o La Religión Pura en Doze Palabras Fieles y Dignas de ser Recibidas de Todos (1699).

Plus tard, ce sera Benjamin Franklin lui-même qui estimera utile et nécessaire d'introduire l'étude de l'espagnol comme matière obligatoire dans l'enseignement publique à l'Université de Pensylvannie qui deviendra, de nos jours, l'un des plus importants centres d'études hispaniques. La liste est longue de ceux qui continuèrent dans ce sillage jusqu'à ce que des hispanistes tels que William Prescott et John Lloyd Stephens, poussés par le besoin de légitimer une identité américaine sur la base de racines liées au continent et non pas marquées par la référence à la culture européenne - lisons derrière ce projet la contreattaque à la latinité préchée par Louis Napoléon-en viennent à asseoir la fierté d'être Américain sur l'héritage préhispanique (!!!): les trésors mayas, aztèques et zapotèques suffisaient à récupérer le passé à l'arrivée des Européens et du coup rachetaient les stigmates dont l'Europe avait marqué les cultures autres que celles du bassin méditerranéen des périodes classiques. C'est ainsi que le passé préhispanique, dont l'auréole s'élargissait de la sorte jusqu'aux territoires du nord, devenait curieusement l'essence de "l'américanité", drapeau identitaire qui, plus curieusement encore, ne tenait pas le moins du monde compte des 
peuples descendant directement de ces cultures, indiens ou métis en tout cas plus autorisés à se réclamer tels. ${ }^{12}$

Pour terminer, je m'aperçois qu'aux questions qui ont guidé ces réflexions (y a-t-il, pour les Canadiens, un référent différent de celui qui renvoie à Amérique=Etats-Unis dans la notion d'Américanité? Quelle place ont occupée dans leur imaginaire les autres peuples du continent? Comment les Québécois, en particulier, perçoivent-ils leur parenté avec les Latinoaméricains? À quoi pensent les autres habitants de l'Amérique lorsqu'ils se disent Américains?), il aurait fallu, pour des raisons de rigueur et de justice vis-à-vis de tous les habitants de ce continent, en ajouter d'autres aussi capitales et pertinentes que je me permets tout juste d'évoquer très brièvement.

D'aucuns penseront que ces commentaires ont passablement simplifié le paysage humain du continent en n'y distinguant que deux grands contingents pouvant se réclamer d'une américanité dont nous avons tenté de relever les différences les plus évidentes. Nous sommes conscients du silence dans lequel nous avons laissé une portion peu négligeable de la population de l'Amérique: les Indiens du nord au sud du continent. Nous aurions dû nous demander également comment perçoivent-ils leur américanité, si jamais ils se sentent Américains? Et encore, peut-on escompter que leur sentiment d'appartenance à ces terres a quelque chose à voir avec ce que nous appelons nos racines métisses? Sachant qu'à l'heure actuelle, les divers groupes d'indiens d'un bout à l'autre de l'Amérique vivent dans des conditions qui couvrent pratiquement tous les degrés du développement et du sous-développement, ce qui suppose de quelque manière le maintien ou la disparition de leurs traditions culturelles, parfois peu compatibles avec les valeurs de la modernité et de la technologie, sachant donc ceci est-il permis d'imaginer que leur conscience identitaire soit comparable à celle des peuples dont ils partagent le territoire?

Les innombrables programmes, menés des deux côtés de l'océan pour la commémoration du 5ème Centenaire de l'arrivée de Christophe Colomb en Amérique, n'ont pas manqué de raviver de vieilles plaies et de susciter autant de questions au sujet de l'identité de ceux qui pouvaient revendiquer des droits d'appartenance à ce Nouveau Monde. Mais les pages de ce livre d'histoire sont encore en train de s'écrire; celles à venir nous obligeront sûrement à poser un

${ }^{12}$ Il y aurait lieu de se demander pourquoi Prescott et Stephens n'ont pas revendiqué directement le patrimoine culturel des peuples amérindiens des Etats-Unis et du Canada — qu'ils ont exterminés, ou du moins réduits et parqués dans de vrais ghettos- pour asseoir et justifier leur "américanité"? Serait-ce parce que l'Indien, réduit au statut de pièce de musée ou de bête de zoo, n'est pas dangereux? Celui qui peut réclamer des droits, exiger d'être respecté et traité en partenaire, celui-là n'existe pas. 
autre regard sur les chapitres que nous avons survolé ici de même que sur ceux que nous vivons en ce début de siècle.

\section{Bibliographie}

ATTALI, Jacques. 1991. 1492. Paris: Fayard.

Beltrán, Rosa. 1996. América sin americanismos. México: UNAM, FFyL.

BISSOONDATH, Neil. 1995. Le marché aux illusions. Montréal: Boréal-Liber.

BouCHARD, Gérard. 2001. Genèse des nations et cultures du Nouveau Monde.

Montréal: Boreal-Compact, $\mathrm{N}^{\circ} 126$.

CHASSAY, Jean-François. 1995. L'ambiguïté américaine. Quebec: XYZ Editeur.

DEPESTRE, René. 1986. Buenos días y adiós a la negritud. Trad. Ofelia GRONLIER. La Habana: Cuadernos Casa de las Américas, No 29.

FUENTES MARES, José. 1984. Génesis del expansionismo americano. México: El Colegio de México.

LeZAMA LimA, José. 1993. La expresión americana. Ed. Irlemar CHIAMPI. México: FCE.

LiTALIEN, Raymonde. 1993. Les explorateurs de l'Amérique du Nord. 14921795. Quebec: Les Éditions du Septentrion.

MORENCY, Jean. 1994. Le mythe amérindien dans les fictions d'Amérique. Quebec: Nuit Blanche Editeur.

MORIN, Michel. 1982. L'Amérique du Nord et la culture. Quebec: Hurtubise $\mathrm{HMH}$.

O'GORMAN, Edmundo. 1984. La invención de América. México: FCE / SEP. (Lecturas Mexicanas, 63)

ORTEGA Y MÉDINA, Juan A. 1987. Imagología del bueno y del mal salvaje. México, UNAM, IIH.

Provencher, Serge. 1977. "Introduction", Faucher De SAINT-MAurice. Contes et récits. Montreal: VLB Éditeur.

PUMAR MARTíNeZ, Carmen. 1991. Españolas en Indias. México: REI.

SIMON, Sherry et al. 1991. Fictions de l'identitaire au Québec. Montréal: XYZ Editeur.

THÉRIEN, Gilles. 1986. "La littérature québécoise, une littérature du tiersmonde?" Voix\&Images, Littérature québécoise, №34. Montréal: UQAM.

Todorov, Tzvetan. 1987. La conquista de América. La cuestión del otro. Trad. Flora BoTTON. México: Siglo XXI.

VV. AA. 1993. La latinidad y su sentido en América Latina. México: UNAM (actes du symposium). 